LM-05K176

January 4, 2006

\title{
Effect of Substrate Surface Defects and Te Dopant Concentration on Crystalline Quality and Electrical Characteristics of AlGaAsSb Epitaxial Layers
}

H Ehsani, N Lewis, G Nichols, L Danielson, M Dashiell, Z Shellenbarger and C Wang

This report was prepared as an account of work sponsored by the United States Government. Neither the United States, nor the United States Department of Energy, nor any of their employees, nor any of their contractors, subcontractors, or their employees, makes any warranty, express or implied, or assumes any legal liability or responsibility for the accuracy, completeness or usefulness of any information, apparatus, product or process disclosed, or represents that its use would not infringe privately owned rights. 


\title{
Effect of Substrate Surface Defects and Te Dopant Concentration on Crystalline Quality and Electrical Characteristics of AlGaAsSb Epitaxial Layers
}

\author{
H. Ehsania) ${ }^{a}$, N. Lewis, G. J. Nichols, L. Danielson, and M. W. Dashiell \\ Lockheed Martin Corp., Schenectady, New York 12301-1072, USA \\ Z. A. Shellenbarger \\ Sarnoff Corp., Princeton, New Jersey 08543, USA \\ C. A. Wang \\ Lincoln Laboratory, Massachusetts Institute of Technology, Lexington, MA 02420-9108, USA
}

\begin{abstract}
The influence of GaSb substrate surface defects such as native oxides on the crystalline quality of epitaxial layers was investigated using transmission electron microscopy (TEM). Cross sectional TEM imaging showed that there are discrete defects at the GaSb-substrate/epilayer interface. Secondary ion mass spectroscopy (SIMS) results revealed high oxygen concentration at the interface, indicating that the defects are likely oxides and presumed to be native oxides since other impurities were not detected. High resolution TEM micrographs showed that the subsequent growth of the epilayer continues beyond the defects without any additional defect generation or propagation. Tellurium doped AlGaAsSb epitaxial layers were grown lattice-matched on GaSb substrates and lattice-mismatched on semiinsulating GaAs substrates by organometallic vapor phase epitaxy. Secondary ion mass spectroscopy and Hall data showed that the ratio of carrier concentration to Te concentration decreases significantly when the carrier concentration increases from $2.5 \times 10^{17} \mathrm{~cm}^{-3}$ to $6.5 \times 10^{17} \mathrm{~cm}^{-3}$. TEM imaging showed that the material with heavily doped Te generates a high density (about $10^{8} \mathrm{~cm}^{2}$ ) of planar defects (stacking fault) located on (111) planes. Most of the Te-related defects originate at the GaSb buffer layer/AlGaAsSb epilayer interface. In addition, discrete precipitates were observed in the heavily doped AlGaAsSb layer.
\end{abstract}


a)e-mail: ehsanha@kapl.gov

Keywords: OMVPE; Interface defect; Te doping; AlGaAsSb; TEM characterization; Hall and SIMS measurements 


\section{Introduction}

There is an increasing interest in the Sb-based materials because of lattice matching to GaSb or InAs substrates. These materials also exhibit a wide bandgap range from infrared to visible light for application in thermophotovoltaics (TPV) ${ }^{(1)}$, optoelectronics ${ }^{(2)}$, and high speed devices ${ }^{(3)}$. In addition, large bandgap AlSb-based compound semiconductors lattice matched to GaSb-based low bandgap materials are promising for confinement of minority carriers (low surface recombination velocity). For high performance devices or confinement layers, high crystalline quality is required for both n-type and p-type materials.

For the growth of InGaAsSb and AlGaAsSb materials, GaSb substrates are often used because of lattice matching. Since the epi-ready (the etching process is performed at the substrate supplier) GaSb substrates is not available, the substrates need to be etched prior to loading into the growth chamber to remove the native defects on the substrates surface. While several chemical etching solutions have been investigated and reported in the literature ${ }^{(4-5)}$, none of them provide satisfactory results. Defects on the substrate surface such as native oxides behave as the nucleation sites for generation of microstructural defects and often have significant impact on the crystalline quality of epitaxial layers. The effect of GaSb surface defects on the crystalline quality of subsequent epitaxial layers has not been investigated in detail. In this study TEM was used to determine the effect of residual defects on the crystalline quality of the epitaxial layers.

One of the issues associated with the epitaxial growth of Sb-based materials is n-type doping. Group IV elements such as Si, Ge, and Sn can be incorporated as n-type or p-type dopants, depending on the growth conditions, relative tetrahedral radius, and electronegativity. In Sb-based materials, group IV elements incorporated in group V sites behave as p-type ${ }^{(6-7)}$. Therefore, the choice for n-type doping is limited to the group VI elements, namely S, Se, and Te. Preliminary studies of S and Se doping in GaSb layers showed that these dopants are not as efficient as Te dopant. To date, Te is 
commonly used as an n-type dopant in most Sb-based materials. However, the effect of Te incorporation on the crystalline quality and carrier concentration in AlGaAsSb has not been fully investigated.

In this paper, the effect of substrate surface defects on the crystalline quality of AlGaAsSb layers is reported. The effects of Te incorporation on the crystalline quality and electrical characteristics of AlGaAsSb layers are investigated. For these investigations, TEM, SIMS, and Hall measurements were used.

\section{Experimental procedure}

AlGaAsSb layers lattice matched to GaSb substrates were grown by Organo-Metallic Vapor Phase Epitaxy (OMVPE) on (100)GaSb substrates, miscut 6 degrees towards (111)B and on semiinsulating (100)GaAs miscut 2 degrees toward (110) in a low pressure vertical rotating disc reactor in a

chamber equipped with a load-lock system ${ }^{(8)}$ (details of the growth conditions was reported in reference 8). The precursors used were Tritertirarylaluminum (TTBAl), Triethylgallium (TEGa), Trimethylantimony (TMSb), 1000-ppm arsine in hydrogen, and 100-ppm diethyltelluride in $\mathrm{H}_{2}$ for ntype dopant. The GaSb substrates were dipped in diluted $\mathrm{HCl}$ for a few minutes to remove the native oxides. The GaAs substrates were dipped in $\mathrm{NH}_{4} \mathrm{OH}$ prior loading into the growth chamber.

Prior to the growth of AlGaAsSb, a thin ( $0.3 \mu$ m thick) GaSb buffer layer was grown on GaSb substrates with the same growth condition as the AlGaAsSb layer.

\section{Results and discussions}

Figure 1 shows a TEM cross-sectional image of the GaSb substrate/GaSb buffer/undoped AlGaAsSb layer stack. The image shows a defect layer along the GaSb-buffer/GaSb-substrate interface. The chemical composition of these interfacial defects was not revealed by energy dispersion x-ray spectroscopy in the TEM system because of the small volume of these defects. To determine the nature of these defects, SIMS analysis was performed. SIMS profiles indicated the presence of high oxygen 
concentration at the GaSb-substrate/GaSb-buffer interface as shown in Figure 2. Therefore, we postulate that the nature of the 3-10 nm sized interfacial defects is likely native oxides since no other constituents besides oxygen and the substrate material were observed. To determine the influence of these defects on the crystalline quality of the GaSb buffer layer, high resolution TEM (HRTEM) was performed. The TEM results showed that the subsequent growth of the GaSb buffer layer continues on top of the defects as a single crystal without generation of any new defects as shown in Figure 3. The continuation of the single crystal growth beyond the defects could be attributed to the lateral growth of GaSb over the defects possibility reducing interfacial strain. The presence of small defects (native oxides) at the interface of the GaSb-substrate/GaSb-buffer layer does not generate dislocations or defects that propagate into the active layer. This result indicates that the growth of a thin GaSb buffer layer on GaSb substrates is important for mitigating the effect of substrate residual native oxides on the crystalline quality of the active layers.

AlGaAsSb materials with about $1 \mathrm{eV}$ bandgap energy (related to $20 \%-25 \%$ Al concentration) were grown lattice matched on GaSb substrates for TEM and SIMS investigation and on lattice mismatched semi-insulating GaAs substrates for Hall measurements. The growth of AlGaAsSb layers was performed on the GaSb buffer layer with the same growth temperature and without any growth interruption. The Al concentration was determined by growing AlGaSb on GaSb substrates, and analyzing the composition of AlGaSb layer using high resolution double axis X-ray diffraction. The aluminum concentration was calculated from the epilayer-substrate X-ray rocking curves peak separation. The arsenic composition was determined by adding arsine to the system and adjusting the arsine flow rate until the rocking curves of AlGaAsSb epilayer and GaSb substrate overlaped (related to 2.0\%-2.5\% As concentration). The background carrier concentration of AlGaAsSb is p-type with the carrier concentration of about $2 \times 10^{17} \mathrm{~cm}^{-3}$. The typical background p-type carrier concentration in the GaSb epitaxial layers grown on GaAs substrates is about $3-4 \times 10^{16} \mathrm{~cm}^{-3}$ which is nearly an order of magnitude less than the background carrier concentration in AlGaAsSb layers. The p-type background 
doping in GaSb is correlated with the antisite defects such as Ga on Sb sites or Ga vacancies complex with antisite defects ${ }^{(9)}$. The higher background doping in AlGaAsSb is attributed to the C incorporation from $\mathrm{Al}$ source ${ }^{(10)}$.

AlGaAsSb layers were grown on GaSb-buffer/GaSb-substrates for TEM and SIMS characterizations and on semi-insulating GaAs substrate/ GaSb-buffer for electrical characterizations. Table 1 shows the Te concentration and carrier concentration in AlGaAsSb layers characterized by SIMS and Hall measurements, respectively (For the SIMS characterization, a Te implanted GaSb standard was used. Since the low and heavily doped layers were compared with the same standard, the relative compositions observed in these samples are reasonable).

Table 1: Comparison of Te doped of two samples with different Te concentration.

\begin{tabular}{|c|c|c|}
\hline Sample & $\begin{array}{c}\text { Electron Concentration }\left(\mathrm{cm}^{-3}\right) \\
\text { Hall Measurement }\end{array}$ & $\begin{array}{l}\text { Te Concentration }\left(\mathrm{cm}^{-3}\right) \\
\text { SIMS Depth Analysis }\end{array}$ \\
\hline AlGaAsSb & $2.5 \times 10^{17}$ & $4.8 \times 10^{17}$ \\
\hline AlGaAsSb & $6.5 \times 10^{17}$ & $1.3 \times 10^{19}$ \\
\hline
\end{tabular}

Comparison of the Hall and SIMS data indicates that the ratio of the carrier concentration to Te concentration in low doped Te concentration is significantly higher than the heavily doped Te (by about an order of magnitude). These results indicate that as the Te concentration increases, the percentage of inactive Te increases. It has been shown that in some Sb-based materials ${ }^{(11)}$, the carrier concentration reaches a saturation level and then declines as the Te concentration increases further. This behavior has been attributed to Te precipitation, formation of chalcogenide particles (due to high reactivity of Te with $\mathrm{Ga}, \mathrm{Al}$, and Sb) as undesirable phases in the epilayer, and donor related deep levels. High Te concentrations might also degrade the crystalline quality of Sb-based materials. We observed that the surface morphology of the AlGaAsSb with high Te concentration was rougher compared with the low 
Te concentration samples. The rougher surface morphology of the heavily doped Te might be attributed to the degradation of the crystalline quality of the AlGaAsSb epitaxial layers.

To determine the effect of Te concentration on crystalline quality of AlGaAsSb epilayers, crosssectional TEM was performed on the heavily and lightly Te-doped samples. Figure 4 shows a TEM image of heavily Te-doped AlGaAsSb with the Te concentration of $1.3 \times 10^{19} \mathrm{~cm}^{-3}$. The analysis of the planar defects showed that they are located on (111) planes. The diffraction contrast is not consistent with dislocations but is consistent with stacking faults. However, we are not certain of the type of stacking fault. This type of defects was only observed in the heavily Te doped samples. The TEM image also shows that most of the planar defects initiated at the GaSb buffer layer/AlGaAsSb interface and propagate through the AlGaAsSb layer. The reason for initiation of these defects at the interface of GaSb-buffer/Te-doped AlGaAsSb layers might be associated with the induction of local stress due to incorporation of high Te concentration at the surface. High Te concentration may cause enough local lattice strain to nucleate planar defects. The influence of high Te concentration on the surface reconstruction and degradation of $\mathrm{GaSb}^{(12)}$ and also on the ordering of $\mathrm{GaInP}^{(13)}$ has been reported. The estimated defect density is in the $10^{8} \mathrm{~cm}^{-2}$ ranges. In contrast, the TEM image of the lightly doped $\left(2.5 \times 10^{17} \mathrm{~cm}^{-3}\right)$ sample contains no defects, as shown in Figure 5. These results suggest that the defects generated in the heavily doped samples are related to the Te incorporation. In the current study small distinct particles (25 nm size) were also observed in the AlGaAsSb epilayer as indicated in Figure 6. The formation of these particles could be due to Te precipitation and formation of undesirable phases. The chemical structure of these defects was not revealed by the EDX in the TEM is due to the small volume of the defects.

\section{Conclusions}

The effect of substrate surface defects (native oxides) on the crystalline quality of AlGaAsSb grown on GaSb substrate by OMVPE has been investigated. TEM imaging results showed that there is a defect layer at the GaSb-substrate/epilayer interface. SIMS results suggested that the interfacial 
defects are mostly native oxides. The data suggests that the effect of substrate surface defects can be suppressed by growing a thin GaSb buffer layer on GaSb substrate. SIMS and Hall data showed that the ratio of active Te concentration over the total Te concentrations decreases rapidly as the Te concentration increases. TEM data showed that the high Te concentration in AlGaAsSb films generates stacking faults, often originating at the GaSb buffer/AlGaAsSb epilayer interface.

Acknowledgements: The authors would like to thank Al Ferguson for the cross-sectional TEM sample preparation. 


\section{References}

[1] C. Hitchcock, R. Gutmann, J. Borrego, H. Ehsani, I. Bhat, M. Freeman, and G. Charache, Thermophotovoltaic Generation of Electricity, AIP Conf. Proc., 401 (1997).

[2] D. H. Chow, R. H. Miles, T. C. Hasenberg, A. R. Kost, Y. H. Zhang, H. L. Dunlap, L. West, Appl. Phys. Lett. 67, 3700 (1995).

[3] K. Taira, K. Funato, F. Nakamura, and H. Kawai, J. Appl. Phys. 69, 4454 (1991).

[4] L.J. Gomez and M.T. Montojo, J. Electrochem. Soc, 136, 1480 (1989).

[5] C.A. Wang, D.A. Shiau, and A. Lin, J. Cryst. Growth, 261, 385 (2004).

[6] H. Ehsani, I. Bhat, R. Gutmann, G. Charache, Appl. Phys. Lett. 69, 3863 (1996).

[7] R. M. Biefeld, B. T. Cunningham, S. R. Kurtz, and J. R. Wendt, Matr. Res. Soc. Symp. Proc. 216, 175 (1991).

[8] Z. Schellenbarger, G. Taylor, R. Martinelli, and J. Carpinelli, $6^{\text {th }}$ AIP Conf. Proc.(2004).

[9] A. G. Milnes and A. K. Polyakov, Solid-State Electron, 36, 803 (1993).

[10] C.A. Wang, J. Cryst. Growth, 170, 725 (1997).

[11] H. Ehsani, I. Bhat, C. Hitchcock, R. J. Gutmann, G. Charache, and M. Freeman, J. Cryst. Growth, 195, 385 (1998).

[12] T. H. Chi, J. A. Ditzenberger, H. S. Luftman, W. T. Tsang, and N. T. Ha, Appl. Phys. Lett. 56, 1688 (1990).

[13] S. W. Jun, G. B. Stringfellow, A. D. Howard, C. M. Fetzer, and J. k. Shurtleff, J. Appl. Phys. 90, 6048 (2001). 


\section{Figures caption}

Figure 1. TEM image showing interface defects at the GaSb buffer layer grown on (001) $6^{\circ}$ off toward (111)B GaSb substrate at $525{ }^{\circ} \mathrm{C}$.

Figure 2. SIMS characterization of an AlGaAsSb epitaxial layer grown on GaSb substrates is shown. High oxygen concentration at the interface of GaSb-substrate/GaSb buffer layer is demonstrated.

Figure 3. High resolution TEM image of the GaSb buffer/GaSb substrate interface showing local interface defects.

Figure 4. TEM image of heavily doped Te in AlGaAsSb epilayer grown on GaSb substrate. Planar defects in the epilayer are mostly nucleated at the interface.

Figure 5. TEM image of lightly doped Te in AlGaAsSb epilayer grown on GaSb substrate. No defects related to Te incorporation are observed.

Figure 6. Small defect particles in the heavily doped Te in AlGaAsSb epilayer grown on lattice matched GaSb is observed. 


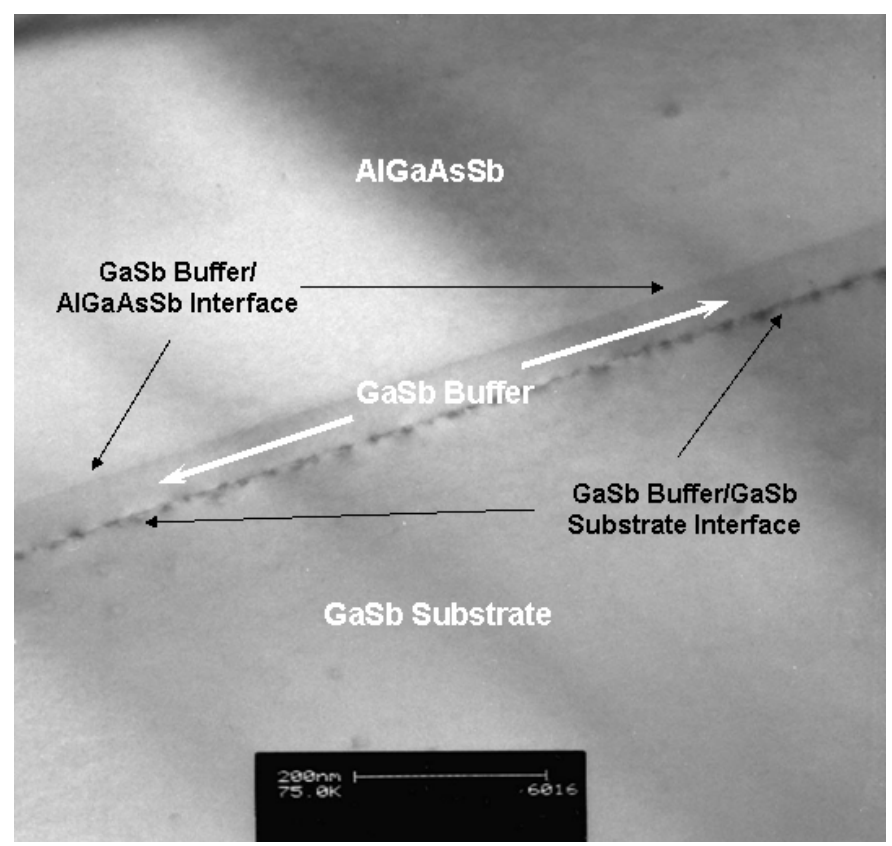

(Figure 1) 


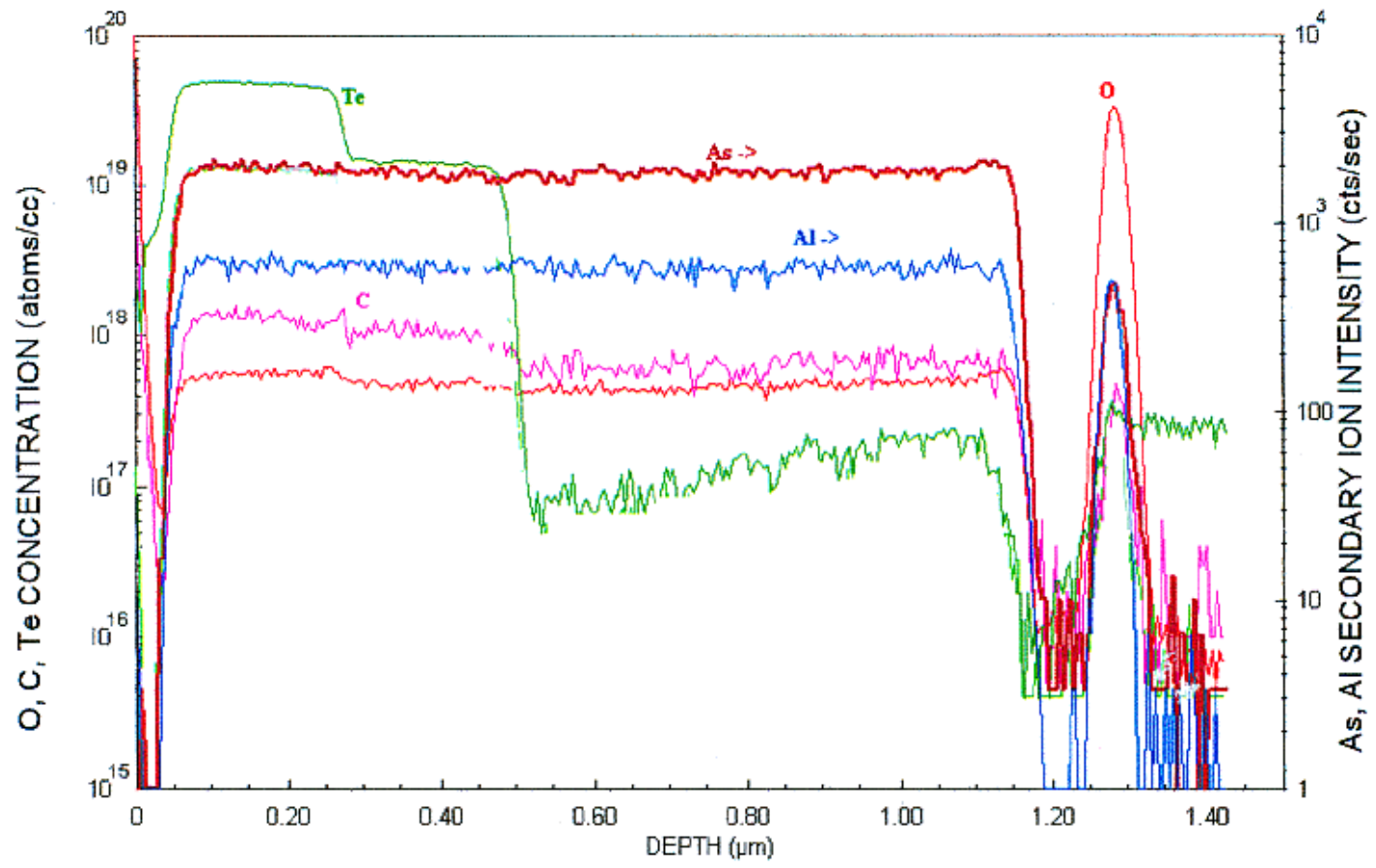

(Figure 2) 


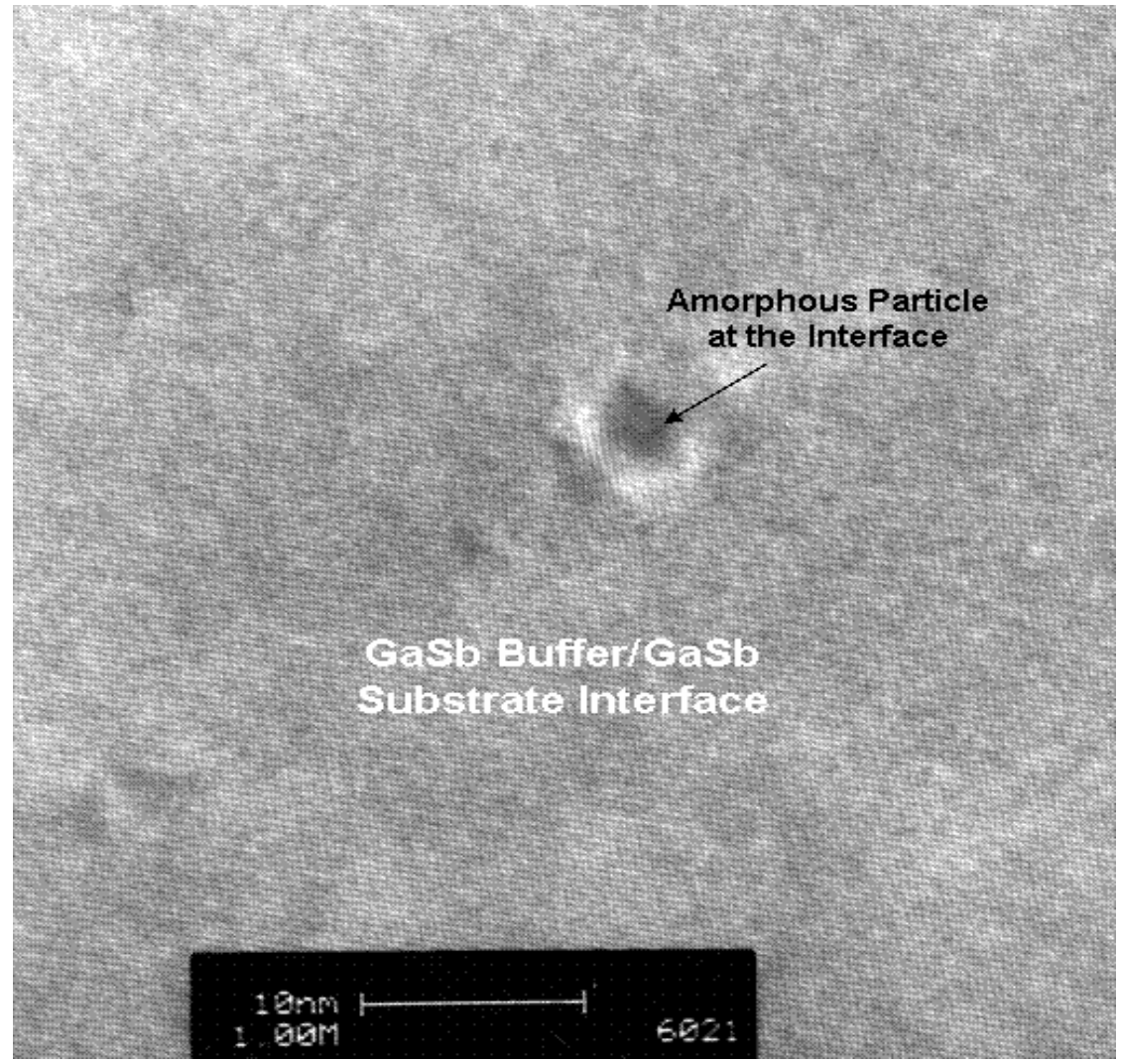

(Figure 3) 


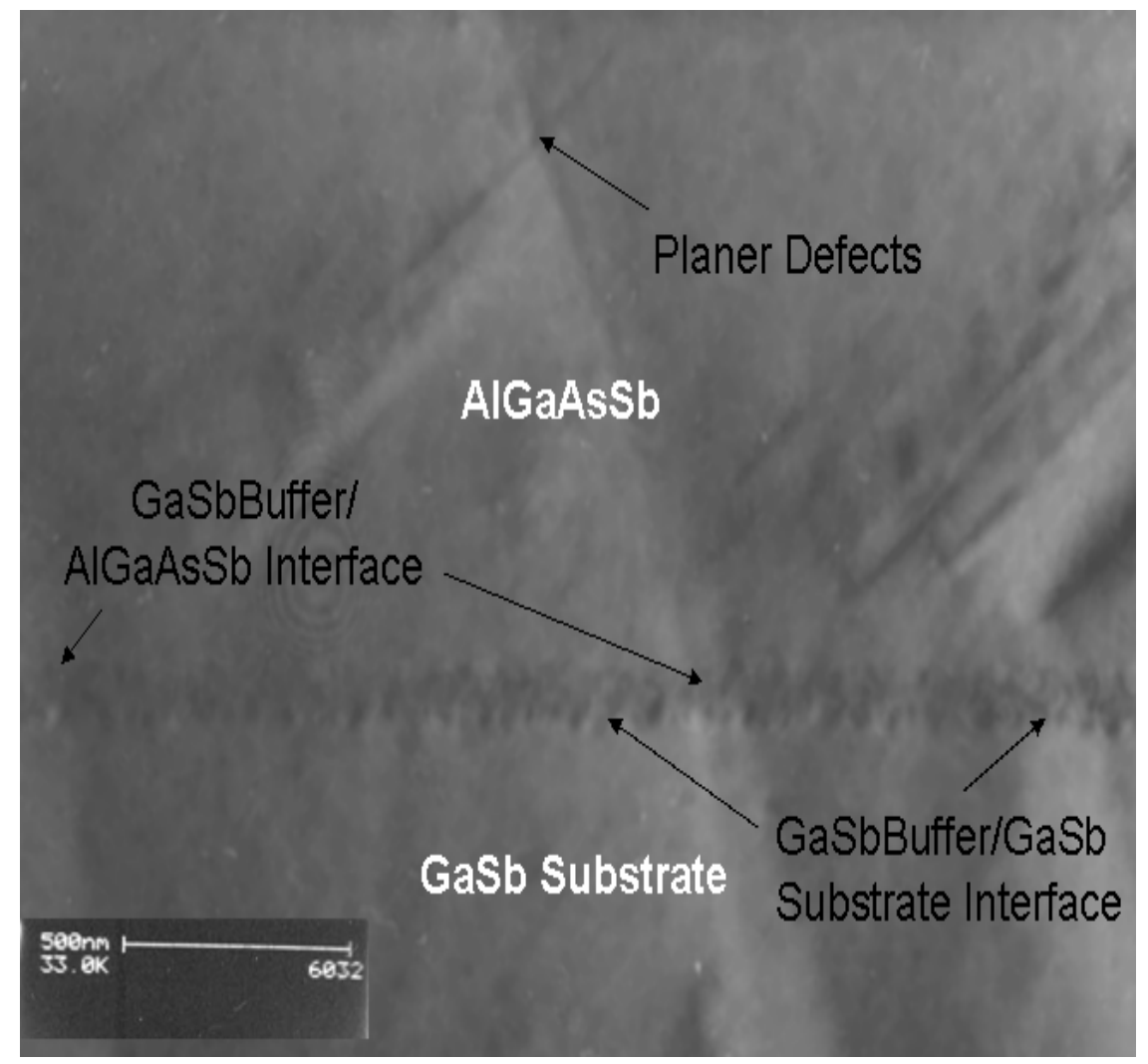

(Figure 4) 


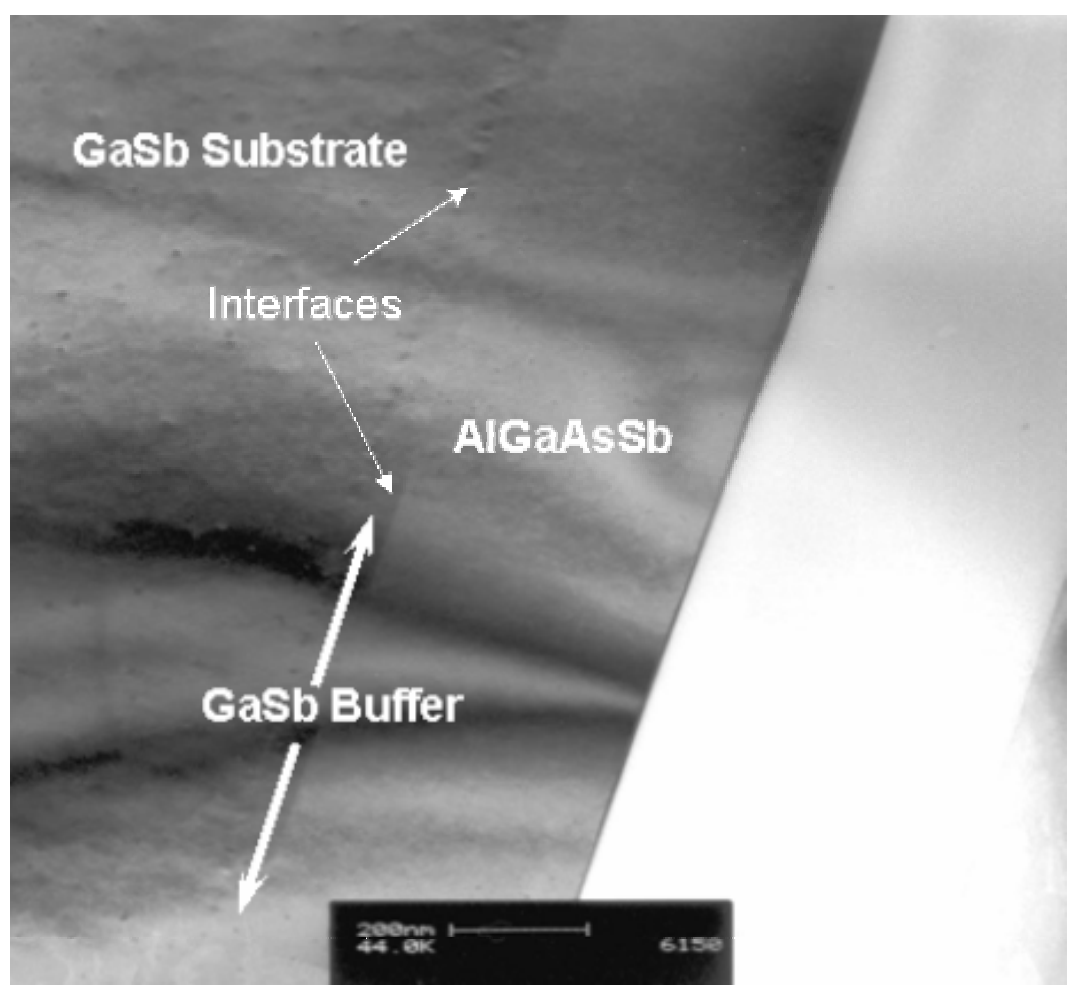

(Figure 5) 


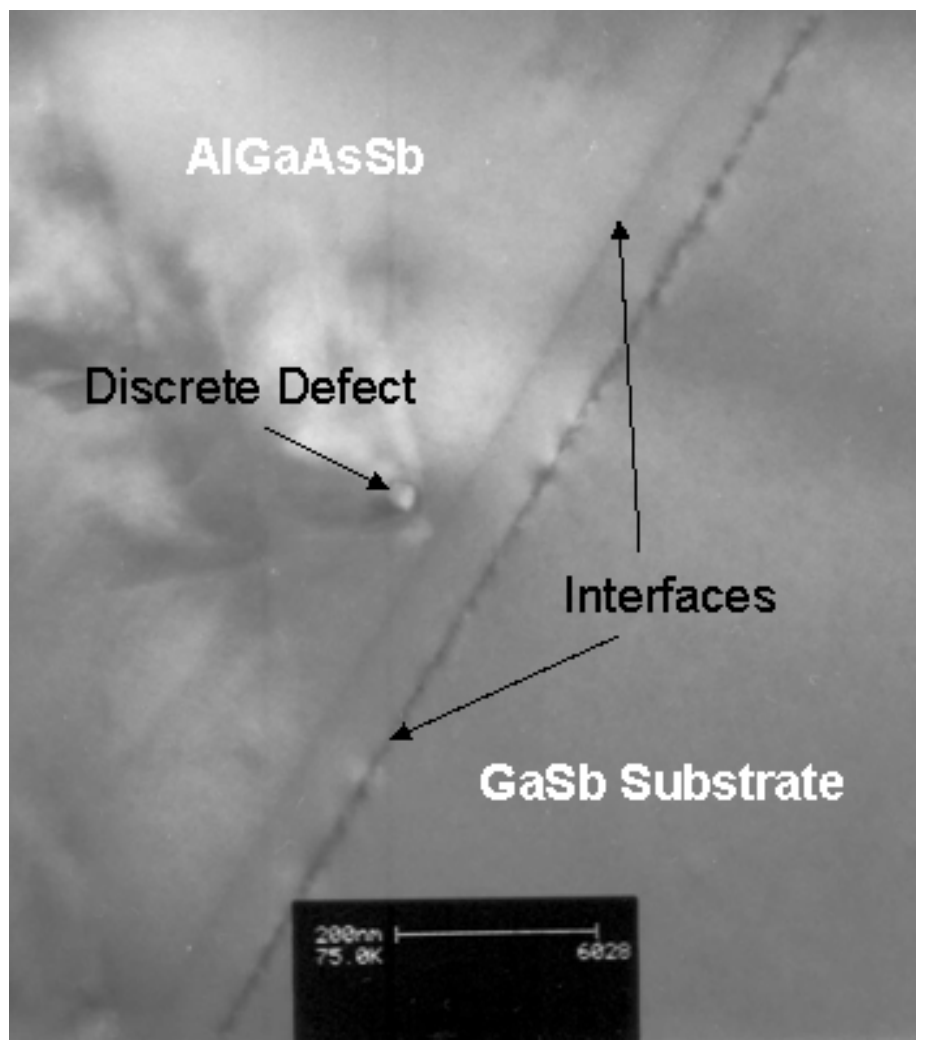

(Figure 6) 